\title{
Mortalidad por defectos al nacimiento en menores de 5 años de edad en México de 1998 a 2006
}

\author{
Javier Valdés-Hernández, Dr en CSC, (1), Sonia Canún-Serrano, MC, (2) \\ Adelmo E Reyes-Pablo, MC,(2) Eduardo Navarrete-Hernández, Tec Est SP.(3)
}

\author{
Valdés-Hernández J, Canún-Serrano S, \\ Reyes-Pablo AE, Navarrete-Hernández E. \\ Mortalidad por defectos al nacimiento en menores \\ de 5 años de edad, México, 1998 a 2006. \\ Salud Publica Mex 2009;5 I:38 I-389.
}

\section{Resumen}

Objetivos.Analizar la mortalidad por defectos al nacimiento (DAN) entre 1998 y 2006. Seleccionar los municipios con alta mortalidad en $<5$ años de edad. Material y métodos. Las fuentes de información fueron los registros de mortalidad de las estadísticas vitales de SSA/INEGI. Se utilizó el Marco Geoestadístico Municipal 2005 del INEGI y el SIGEPI para el análisis espacial. Los criterios de selección fueron los municipios con percentiles de 80 y mayores de las defunciones por DAN. Resultados. Los decesos disminuyeron $8 \%$ en el periodo de 1998 a 2006 y las tasas $20 \%$. Una proporción de 42.57 a $48 \%$ de las muertes se debe a defectos del sistema circulatorio y 13.69 a $19.39 \%$ del sistema nervioso; las primeras se incrementaron $4 \%$ y las segundas descendieron $32 \%$. Hasta $80 \%$ o más ocurre en $<5$ años y la tasa en este grupo decreció $8.63 \%$. Un número de I 025 (4I.82\%) municipios es prioritario, $104(10.14 \%)$ de alta y $102(9.95 \%)$ de muy alta prioridad. En ellos se produce $66 \%$ de las muertes en $<5$ años. Discusión. Las intervenciones para reducir la mortalidad por DAN deben focalizarse en los menores de I año (75\%) y en $8.4 \%$ de los municipios con muy alta prioridad, ya que concentran $66 \%$ de los decesos.

Palabras clave: defectos congénitos; análisis de área pequeña; prioridades en salud; México
Valdés-Hernández J, Canún-Serrano $\mathrm{S}$, Reyes-Pablo AE, Navarrete-Hernández E. Birth defects mortality in five-year-old minors of age, Mexico, 1998-2006.

Salud Publica Mex 2009;5 I:38I-389.

\begin{abstract}
Objective. To analyze mortality due to birth defects from 1998-2006.To select municipalities with high mortality among children under 5 years of age. Material and Methods. The source of information was mortality records from vital statistics collected by SSA/INEGI.We used the 2005 Municipal Geostatistical Framework by INEGI and SIGEPI for the spatial analysis. The selection criteria were municipalities with $80 \%$ and over of deaths due to birth defects. Results. Deaths diminished $8 \%$ during 1998-2006 and rates decreased 20\%.A total of $42.57 \%-48 \%$ of deaths are due to circulatory system defects and $13.69 \%$ - $19.39 \%$ are due to the nervous system; the former rose $4 \%$ and the latter fell $32 \%$. Eighty percent or more occur in children under 5 years and the rate in this group fell $8.63 \%$.A total of I $025(41.82 \%)$ municipalities are priorities, $104(10.14 \%)$ are high and $102(9.95 \%)$ are very high priorities, where $66 \%$ of deaths occur among children under 5 years old. Discussion. The interventions to decrease mortality due to birth defects should be directed towards one-year-old children (75\%) and towards $8.4 \%$ of the municipalities that are a very high priority, since they represent $66 \%$ of the deaths.
\end{abstract}

Key words: congenital defects; small-area analysis; priorities health; Mexico

(I) Dirección General de Epidemiología, Secretaría de Salud. México

(2) Hospital General Dr. Manuel Gea González. México

(3) Coordinación de Vigilancia Epidemiológica y Apoyo en Contingencias, Instituto Mexicano del Seguro Social. México 
U na de las funciones de la epidemiología es la identificación de poblaciones con alto riesgo de sufrir daños a la salud, de acuerdo con características de tiempo, lugar y persona, con la finalidad de priorizar áreas, acciones y recursos. En las últimas tres décadas se ha incrementado el empleo de los estudios espaciales con estos propósitos, entre otros más. ${ }^{1}$ En México, los estudios espaciales son escasos y se han enfocado en varias áreas de la salud. ${ }^{2-5}$ Se ubican dentro del diseño de investigación epidemiológica "ecológico". ${ }^{6}$ En la actualidad existe una gran disponibilidad de métodos estadísticos y sistemas de información geográfica (SIG) que facilitan su uso.,8 Sin embargo, la problemática subyacente a estos estudios continúa en términos de la falacia ecológica y se han agregado nuevos retos, como el tamaño de la escala y los límites geográficos y su relación con la variable en estudio o el problema de los números demasiado pequeños, que pueden ocasionar inestabilidad en las tasas, o en extremo grandes y capaces de ocultar variaciones locales de consideración. ${ }^{9}$

El objetivo de los estudios espaciales con fines de detección de áreas de alto riesgo es describir y analizar los datos en salud mediante mapas y determinar si esta distribución adopta ciertas características de aglomeración (patrón espacial) o no (forma aleatoria). ${ }^{10}$ Para resolver el problema de dispersión por inestabilidad en las tasas, se ha propuesto su suavizamiento a través de modelos estadísticos bayesianos. No obstante, el uso exclusivo de las tasas estandarizadas para identificar áreas de riesgo puede excluir a aquellas que proporcionan mayor número de casos, lo cual puede no ser lo idóneo.

En esta publicación se propone usar varios criterios para la selección de municipios prioritarios de muertes por defectos al nacimiento (DAN): la frecuencia absoluta de las defunciones, el suavizamiento de la frecuencia absoluta y las tasas estandarizadas de mortalidad. La Norma Oficial Mexicana define como DAN “...cualquier anormalidad del desarrollo anatomofuncional, del crecimiento/maduración y metabólico, presente al nacimiento, notoria o latente, que interfiera la correcta adaptación del individuo al medio extrauterino en los aspectos biológicos, psíquicos y sociales, que sean capaces o no de ocasionar la muerte o la discapacidad para crecer y desarrollarse en las mejores condiciones, en alguna etapa del ciclo vital." ${ }^{11}$ En México, los DAN figuraron dentro de las 10 primeras causas de mortalidad en niños menores de 14 años de edad en los años 2000 a 2005 y 2007 y las principales afecciones fueron las siguientes: malformaciones congénitas del corazón, anencefalia y anomalías similares, síndrome de Down, espina bífida, defectos de la pared abdominal, fístula traqueoesofágica, atresia y estenosis esofágica y paladar hendido. ${ }^{12}$
Algunos DAN pueden prevenirse, diagnosticarse y atenderse oportunamente si se le otorga a la madre atención médica de calidad antes, durante el embarazo y al momento de la resolución obstétrica; de esa forma, el neonato tendrá mayores posibilidades de una mejor condición de vida. En México se ha establecido como medida de prevención que toda mujer en edad reproductiva consuma una ingesta diaria de ácido fólico de $400 \mu \mathrm{g} /$ día o $0.4 \mathrm{mg}$, especialmente durante la etapa periconcepcional (tres meses antes del embarazo y hasta la semana 12 de la gestación). ${ }^{11}$

Según Emery y Rimoin, las causas de los DAN pueden ser: a) multifactoriales: interacción entre susceptibilidad genética e influencia ambiental; b) cromosómicas: adición o pérdida (completa o parcial) de un cromosoma; c) mendelianas; y d) ambientales: teratogénesis. ${ }^{13}$

Jorde y colaboradores refieren que los defectos congénitos son una causa notoria de mortalidad y morbilidad infantil y señalan que la frecuencia de malformaciones graves que se diagnostican durante el periodo neonatal es de 2 a 3\%, con incremento a 3 a $4 \%$ hacia la edad de 1 año. ${ }^{14}$ En México, según Morales y colegas, la frecuencia de DAN externos en recién nacidos vivos (RNV) es de $2 \%$ y aumenta a $12 \%$ en óbitos; en caso de incluir los DAN internos, las frecuencias se elevan a 2.5\% en RNV y 3.5\% si se consideran ambos, RNV y óbitos. ${ }^{15}$ En 1983, Hernández y colaboradores notificaron 12.8 por 1000 RNV (1.3\%) de DAN. ${ }^{16}$ En 1984, Canún y colegas informaron $3.3 \%$, con $1.2 \%$ que correspondió a malformaciones mayores. ${ }^{17}$ En 1990, Canún y colaboradores registraron $2.5 \%$ de niños con malformaciones en $\mathrm{RNV}$, con $1.8 \%$ para las malformaciones mayores; en abortos espontáneos, hasta $61.5 \%$ presentaba malformaciones $y$, en el caso de los óbitos, la cifra fue de $8.8 \%$, en su mayor parte $(6.7 \%)$ defectos de cierre del tubo neural. ${ }^{18}$ Arredondo de Arreola, en un estudio retrospectivo publicado en 1990, encontró $2.31 \%$ de RNV con malformaciones congénitas. ${ }^{19}$ Fernández y Hernández notificaron $4 \%$ de DAN en el periodo 2001-2004. ${ }^{20}$ Aguinaga y colegas informaron en 2005 una cifra de 3.6\% de DAN.$^{21}$ Vega y Vizzuett encontraron en el mismo año 3.1\% de malformaciones congénitas. ${ }^{22}$ Otros estudios resaltan la importancia que tienen los DAN como causa de mortalidad neonatal temprana y perinatal..$^{23,24}$

Los factores de riesgo establecidos para DAN son: a) edad materna mayor de 35 años; b) edad paterna mayor de $35,{ }^{14,25} \mathrm{c}$ ) antecedentes familiares o reproductivos de DAN; d) padres con enfermedades genéticamente determinadas; $\mathrm{e}$ ) enfermedades maternas; f) exposición a agentes teratógenos; g) consanguinidad; y h) deficiencia de AF periconcepcional. ${ }^{26}$

El objetivo de este trabajo es analizar la tendencia de la mortalidad por DAN en la República mexicana 
durante el periodo de 1998 a 2006, con énfasis en los menores de 5 años de edad, e identificar los municipios con alta prioridad de intervención.

\section{Material y métodos}

La unidad de observación fue el municipio. Se obtuvieron las defunciones por edad de las bases de datos de mortalidad 1998-2006 de la Secretaría de Salud, ${ }^{27}$ con códigos Q00-Q99, correspondientes al Capítulo XVII de la Clasificación Estadística Internacional de Enfermedades y Problemas Relacionados con la Salud. Décima Revisión, que incluyen malformaciones congénitas, deformidades y anomalías cromosómicas. ${ }^{28}$ Para la mortalidad se tomó en cuenta la variable municipio de residencia habitual del fallecimiento. Los datos de población por edad se obtuvieron del censo de población y vivienda del año 2000 y los conteos de población y vivienda de 1995 y $2005 ; ;^{29}$ la de los años intermedios del periodo 1998-2006 se obtuvo en hojas de cálculo de Excel con la técnica de crecimiento lineal. Para la población menor de 1 año y de 1 a 4 años de edad, se aplicó el porcentaje de distribución del Consejo Nacional de Población a nivel nacional para esos años. ${ }^{30}$ Para estandarizar tasas se utilizó la estructura de edad por quinquenios de la población calculada para 1998. Veintiséis (1.06\%) de los municipios no contaron con información; en éstos se interpolaron las defunciones o tasas tras calcular la media de los valores obtenidos en los municipios vecinos.

Con la finalidad de disminuir el problema de heterogeneidad de tasas, propio de los estudios en áreas pequeñas, se utilizaron los siguientes métodos de estabilización: la acumulación de la información de los años estudiados (1998-2006), la estandarización de las tasas por el método directo y el suavizamiento local con la técnica de media móvil con una distancia en vecindad de 100 kilómetros con la finalidad de trazar un patrón espacial. Se empleó la cartografía del Marco Geoestadístico Municipal 2005 del INEGI. ${ }^{31}$ Para el suavizamiento espacial y el mapeo de la información se utilizó el SIGEPI, versión, 3.1. ${ }^{*}$ La estandarización suaviza la tasa con inclinación hacia las áreas de mayor población, mientras que el procedimiento espacial afecta en particular a las tasas extremadamente altas o bajas, ${ }^{32}$ y el resultado es su aglomeración, según vecindad de valores altos o bajos. ${ }^{33-39}$

Para seleccionar los municipios se realizó primero la estratificación de las frecuencias mediante percentiles

\footnotetext{
* OPS. Sistema de Información Geográfica en Epidemiología (SIGEPI), versión 3.1. Washington, EUA 2005.
}

y se eligieron los que se encontraban en el percentil 80 o mayor de las siguientes distribuciones: a) frecuencia absoluta de defunciones; b) frecuencia suavizada de las cifras absolutas de defunciones; y c) tasas estandarizadas de mortalidad suavizadas. A continuación, los municipios prioritarios se dividieron en 10 estratos en relación con percentiles (los incluidos en el estrato 80 a 89 se consideraron de alta prioridad y aquéllos del estrato 90 o mayor se consideraron de muy alta prioridad). El presente informe se extrajo de una investigación independiente $\mathrm{y}$, dado que no se obtuvo información directa de los individuos, no se requirió consentimiento informado.

\section{Resultados}

\section{Tendencia de la mortalidad por defectos al nacimiento, I 998-2006}

Las defunciones por DAN han tenido una disminución en frecuencias absolutas y relativas durante el periodo de 1998 a 2006. En el cuadro I se observa que en 1998 se registraron 10120 defunciones y 9327 en 2006, lo que representa un descenso de $8 \%$. También se advierte que las tasas ajustadas por edad registran un descenso de 20\%, al pasar de 21.52 en 1998 a 17.32 en el año de 2006. $\mathrm{Al}$ observar el comportamiento anual en el cuadro I se reconoce que los números absolutos de defunciones por defectos al nacimiento disminuyen de 1998 a 2003, si bien de 2004 en adelante existe un ligero aumento que no logra modificar la distribución porcentual ni la tasa de mortalidad estandarizada, que se mantienen casi inalteradas en un poco más de $1.87 \%$ y de 17 defunciones por cada 100000 habitantes.

El análisis por causa de DAN destaca que de todas las muertes en el periodo, casi la mitad (42.57 a 48\%) se debió a defectos en el sistema circulatorio (SC) y que por defectos en el sistema nervioso (SN) el porcentaje osciló entre 13.6 y $19.39 \%$; al considerar ambas se obtienen $56.26 \%$ y $67.39 \%$ del total. El examen revela también un descenso de $32 \%$ del número de defunciones por defectos en el SN: en 1998 hubo 1962 muertes y en 2006 descendió a 1302 ; dicho cambio se refleja también en un decremento de $28 \%$ de la proporción de muertes por este tipo de defectos respecto de todas las muertes por DAN, al pasar de un porcentaje de $19.39 \%$ en el primer año a uno de 13.96\% en el último año. En el año 2006 se identifica un leve aumento de las defunciones por defectos en el SN, aunque resalta en mayor medida la tendencia a disminuir. Por el contrario, las defunciones debidas a defectos del SC registran un leve ascenso de 4\%; en 1998 se registraron 4308 muertes y 4460 en 2006; respecto de la proporción, ésta varió de 42.57\% en 1998 a 47.82\% en 
2006, con un incremento de $12 \%$. Las defunciones por otros tipos de DAN variaron en escasa medida, de 3850 en el año base a 3565 en el último año, con un descenso de 7\%; por su parte, la proporción apenas si cambió durante el periodo (datos no incluidos en el cuadro I).
El cuadro II destaca la distribución de muertes y tasas de mortalidad por DAN de acuerdo con los grupos de edad. En referencia al número total de defunciones, más de $72 \%$ se registra en el grupo de menores de 1 año de edad, y entre 82.15 y $85.16 \%$ en los menores de 5 años

Cuadro I

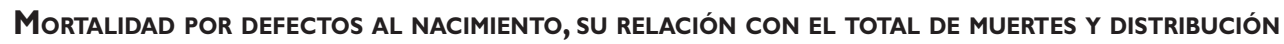
POR GRUPOS DE CAUSAS. MÉXICO, 1998-2006

\begin{tabular}{|c|c|c|c|c|c|c|c|c|c|}
\hline \multirow[b]{3}{*}{ Año } & \multicolumn{3}{|c|}{ Defunciones } & \multicolumn{2}{|c|}{ Tasa* } & \multicolumn{4}{|c|}{ Malformaciones congénitas } \\
\hline & \multirow[b]{2}{*}{ Total } & \multirow{2}{*}{$\begin{array}{l}\text { Defectos al } \\
\text { nacimiento }\end{array}$} & \multirow[b]{2}{*}{$\%$} & \multirow[b]{2}{*}{ General } & \multirow{2}{*}{$\begin{array}{l}\text { Defectos al } \\
\text { nacimiento }\end{array}$} & \multicolumn{2}{|c|}{ Sistema nervioso $0^{\ddagger}$} & \multicolumn{2}{|c|}{ Sistema Circulatorio } \\
\hline & & & & & & Def. & $\%$ & Def. & $\%$ \\
\hline 1998 & 444,665 & 10,120 & 2.28 & 472.15 & 21.52 & 1,962 & 19.39 & 4,308 & 42.57 \\
\hline 1999 & 443,950 & 9,714 & 2.19 & 429.99 & 20.25 & 1,759 & 18.11 & 4,219 & 43.43 \\
\hline 2000 & 437,667 & 9,572 & 2.19 & 413.72 & 19.61 & 1,500 & 15.67 & $4,|7|$ & 43.58 \\
\hline 2001 & 443,127 & 9,178 & 2.07 & 405.64 & 18.47 & 1,377 & 15.00 & 4,163 & 45.36 \\
\hline 2002 & 459,687 & 9,162 & 1.99 & 407.77 & 18.12 & 1,318 & 14.39 & 4,269 & 46.59 \\
\hline 2003 & 472,140 & 8,881 & 1.88 & 405.23 & 17.24 & 1,279 & 14.40 & 4,206 & 47.36 \\
\hline 2004 & 473,417 & 9,058 & 1.91 & 394.88 & 17.27 & 1,283 & 14.16 & 4,328 & 47.78 \\
\hline 2005 & 495,240 & 9,255 & 1.87 & 401.40 & 17.40 & 1,267 & 13.69 & 4,442 & 48.00 \\
\hline 2006 & $494,47 \mid$ & 9,327 & 1.89 & 394.35 & 17.32 & 1,302 & 13.96 & 4,460 & 47.82 \\
\hline $\begin{array}{l}\text { Cambio } \\
1998-2006\end{array}$ & 11.20 & -7.84 & -17.11 & -16.48 & -19.52 & -33.64 & -28.00 & 3.53 & 12.33 \\
\hline
\end{tabular}

Fuentes: Secretaria de Salud. Instituto Nacional de Estadística, Geografía e Informática.

Consejo Nacional de Población.

Cuadro II

DISTRIBUCIÓN PORCENTUAL DE LAS MUERTES POR DEFECTOS AL NACIMIENTO SEGÚN GRUPOS DE EDAD SEleccionados Y TASAS DE MORTALIDAD AJUSTADAs. MÉXICO, 1998-2006

\begin{tabular}{|c|c|c|c|c|c|c|c|c|c|}
\hline \multirow[b]{3}{*}{ Año } & \multicolumn{6}{|c|}{ Grupos de edad } & \multirow{2}{*}{\multicolumn{3}{|c|}{ Tasas ajustadas* }} \\
\hline & \multicolumn{2}{|c|}{ Menor de I año } & \multicolumn{2}{|c|}{ I a 4 años } & \multicolumn{2}{|c|}{ Menores de 5 años } & & & \\
\hline & Def. & $\%$ & Def. & $\%$ & Def. & $\%$ & $<$ l año & I a 4 años & $<5$ años \\
\hline 1998 & 7,612 & 75.22 & $\mathrm{I}, 004$ & 9.92 & 8,616 & 85.14 & 8.13 & 1.07 & 9.20 \\
\hline 1999 & 7,346 & 75.62 & 926 & 9.53 & 8,272 & 85.16 & 7.86 & 0.99 & 8.85 \\
\hline 2000 & 7,212 & 75.34 & 856 & 8.94 & 8,068 & 84.29 & 7.73 & 0.92 & 8.64 \\
\hline 2001 & 6,787 & 73.95 & 836 & 9.11 & 7,623 & 83.06 & 7.36 & 0.90 & 8.27 \\
\hline 2002 & 6,756 & 73.74 & 856 & 9.34 & 7,612 & 83.08 & 7.35 & 0.93 & 8.29 \\
\hline 2003 & 6,450 & 72.63 & 894 & 10.07 & 7,344 & 82.69 & 7.04 & 0.99 & 8.03 \\
\hline 2004 & 6,553 & 72.34 & 900 & 9.94 & 7,453 & 82.28 & 7.18 & 1.00 & 8.18 \\
\hline 2005 & 6,694 & 72.33 & 909 & 9.82 & 7,603 & 82.15 & 7.37 & 1.02 & 8.40 \\
\hline 2006 & 6,834 & 73.27 & 829 & 8.89 & 7,663 & 82.16 & 7.48 & 0.93 & 8.41 \\
\hline $\begin{array}{l}\text { Cambio } \\
\text { 1998-2006 }\end{array}$ & -10.22 & -2.59 & -17.43 & -10.38 & 11.06 & -3.50 & -8.00 & -13.08 & -8.59 \\
\hline
\end{tabular}

* Por 100000 habitantes

Fuente: Secretaria de Salud. Instituto Nacional de Estadística, Geografía e Informática. Consejo Nacional de Población. 
de edad. En los dos grupos de edad se reconoce un descenso de las cifras absolutas y porcentuales de muertes en el periodo, mucho más pronunciado para el último grupo de edad; en cambio, en los menores de 1 año el porcentaje de descenso es de $10 \%$ en las defunciones y de 3\% en el porcentaje; en el grupo de 1 a 4 años, el decremento fue de 17 y $10 \%$, respectivamente. En cuanto a la tasa de mortalidad, el comportamiento del descenso es igual: mayor en el grupo de 1 a 4 años (13\%) que en el grupo de menos de 1 año de edad (8\%).

\section{Municipios prioritarios en mortalidad por DAN en menores de 5 años de edad}

Se trabajó con un total de 70111 defunciones por DAN en el grupo de menos de 5 años durante el periodo de 1998 a 2006 y con 2451 municipios. Un total de 143 defunciones $(0.20 \%)$ en el periodo no tuvo registro del municipio de residencia habitual. La figura 1 muestra la distribución espacial de las frecuencias absolutas y tasas estandarizadas de mortalidad sin suavizar (a)
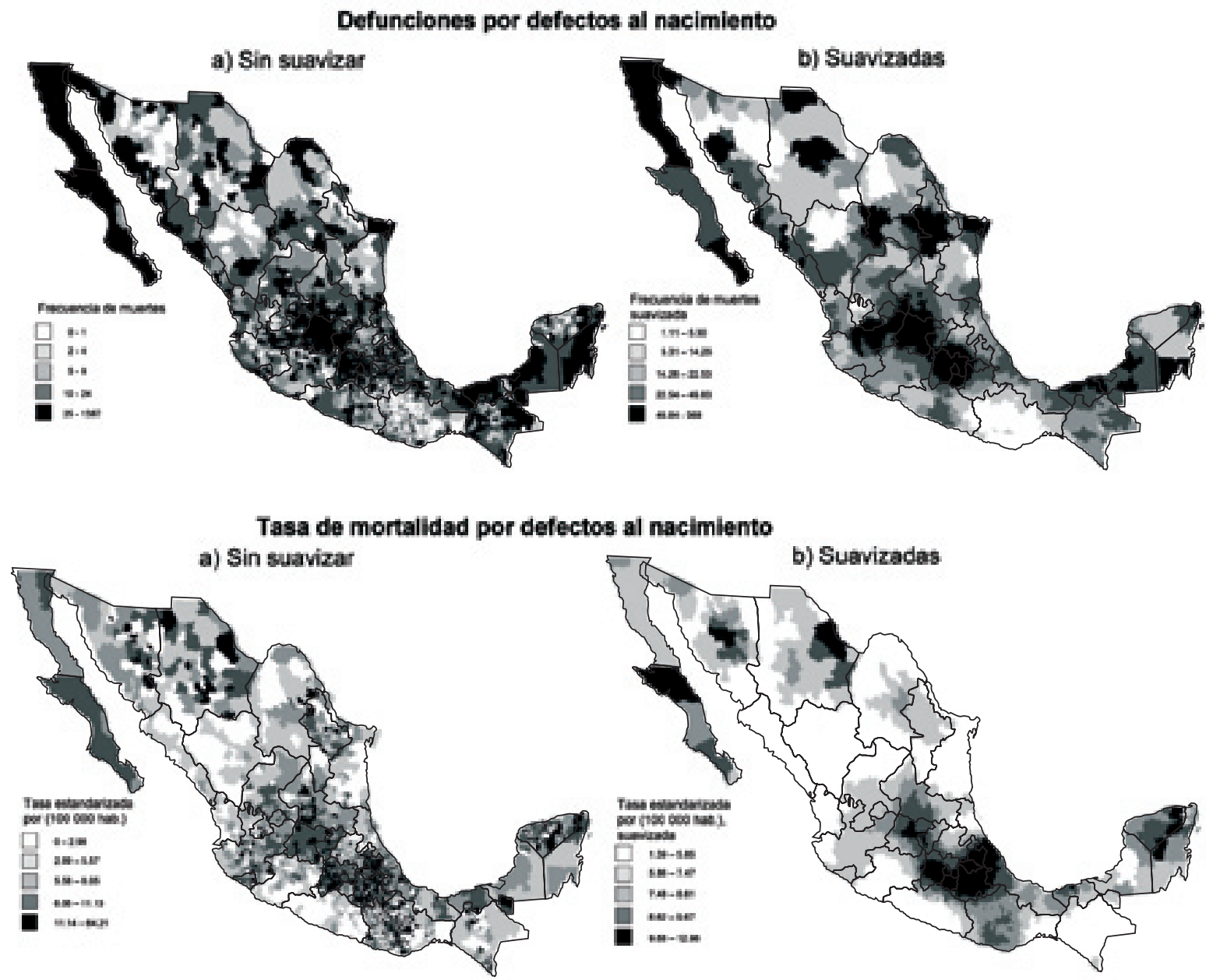

Fuente: Elaboración propia con base en: Mortalidad, bases de datos dinámicas de la SSa y Censo de Población y Vivienda de 2000 y Conteos de Población de 1995 y 2004. INEGI

Figura I. Defunciones y tasa de mortalidad ajustada por defectos al nacimiento en menores de 5 años de edad. Méxıco 1998-2006 
comparada con la distribución espacial de las frecuencias absolutas y tasas de mortalidad suavizadas (b). Las imágenes suavizadas reflejan un patrón espacial mejor delimitado que el moteado de los estimadores sin suavizamiento. Hay que destacar que la amplitud entre los valores mínimos y los máximos se ha reducido con el suavizamiento de los datos: en el caso de las defunciones de 0 a 1387 sin suavizar y de 1.11 a 368 con suavizamiento; $y$ en las tasas estandarizadas de 0 a 64.21 sin suavizar y de 1.39 a 12.96 con suavizamiento. Las áreas totalmente negras de los mapas corresponden a los municipios dentro del percentil 80 o mayor de las distribuciones de frecuencias y se incluyeron como prioritarios. La sobreposición de estas áreas proporcionó como resultado final la conformación de la figura 2, con la integración de los tres criterios empleados.

Del total de los municipios, $1025(41.82 \%)$ se consideraron prioritarios (figura 2); de éstos, 104 (10.14\%) se clasificaron de alta prioridad y $102(9.95 \%)$ de muy alta prioridad. Respecto del total de defunciones, $89.32 \%$
(62 625) acaecieron en los municipios prioritarios, mientras que de esta última cifra, $60.98 \%$ de las muertes ocurrió en los municipios de muy alta prioridad y $15.02 \%$ en los municipios de alta prioridad, lo que suma $76 \%$ entre ambas categorías, lo cual representa $66 \%$ si se considera el total de las defunciones; esto señala una alta concentración de muertes de menores de 5 años por DAN en apenas $8.4 \%$ de los municipios.

La figura 2 muestra que los municipios prioritarios se encuentran localizados en grandes aglomeraciones y distribuidos en el país: ambas entidades californianas, zona costera del Océano Pacífico norte y Golfo de California, frontera con Estados Unidos de América, zonas metropolitanas de la Ciudad de México, Guadalajara y Monterrey y la zona costera de Campeche, Tabasco y Quintana Roo; mientras que los municipios con prioridad alta y muy alta se localizan sobre todo en el centro y norte de la República. El cuadro III enlista los municipios de muy alta y alta prioridad por cada estado.

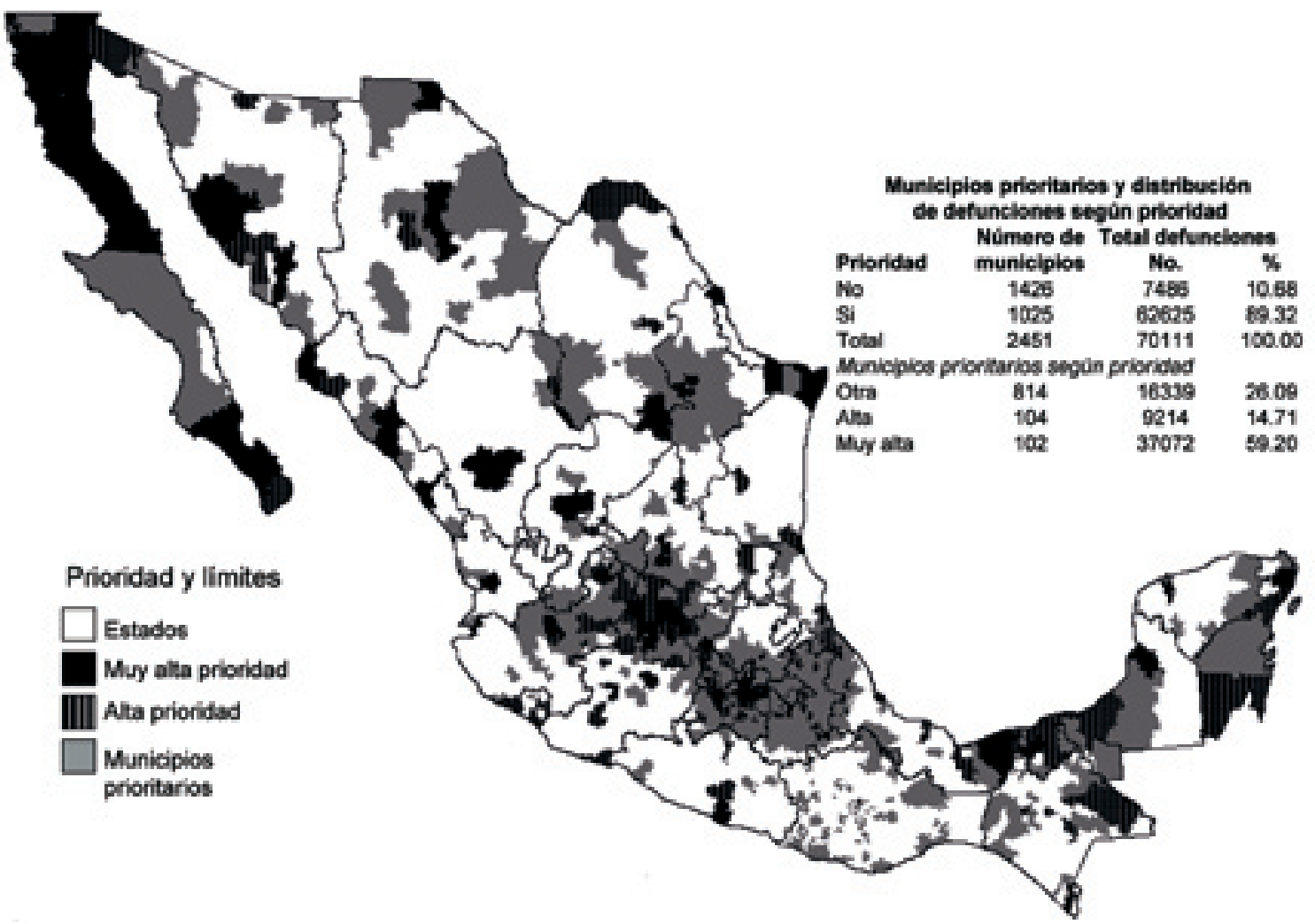

Fuente: Elaboración propia con base en criterios de prioridad y Mortalidad, bases de datos dinámicas de la SSa y Censo de Población y Vivienda de 2000 y Conteos de Población de 1995 y 2004. INEGI

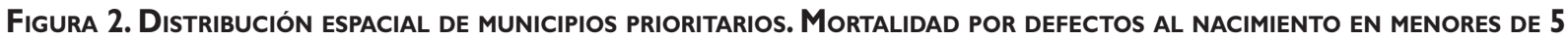
AÑos. MÉxico 1998-2006 


\section{Cuadro III}

RELACIÓN DE MUNICIPIOS POR ENTIDAD FEDERATIVA CON MUY ALTA Y ALTA PRIORIDAD DE MORTALIDAD POR DEFECTOS AL NACIMIENTO EN MENORES de 5 AÑos de EDAD. MÉXICO, 1998-2006

\begin{tabular}{|c|c|c|c|c|c|c|c|c|}
\hline & \multicolumn{2}{|c|}{ Prioridad } & \multicolumn{3}{|c|}{ Prioridad } & \multicolumn{3}{|c|}{ Prioridad } \\
\hline Ent. Fed. & Muy Alta & Alta & Ent. Fed. & Muy Alta & Alta & Ent. Fed. & Muy Alta & Alta \\
\hline Ags. & Ags. & Jesús Maria & Hgo. & Apan & Tulancingo & \multirow[t]{6}{*}{ Pue. } & & Tepeaca \\
\hline \multirow[t]{3}{*}{ BC } & Ensenada & & \multirow[t]{6}{*}{ Jal. } & \multirow{6}{*}{$\begin{array}{l}\text { Guadalajara } \\
\text { Pto.Vallarta } \\
\text { Tlaquepaque } \\
\text { Tonalá } \\
\text { Zapopan }\end{array}$} & \multirow{6}{*}{$\begin{array}{l}\text { Arandas } \\
\text { Lagos de Mor. } \\
\text { Ocotlán } \\
\text { El Salto } \\
\text { Tepetitlán } \\
\text { Tlajomulco }\end{array}$} & & & Teziutlán \\
\hline & Mexicali & & & & & & & Tlacotepec BJ \\
\hline & Tijuana & & & & & & & Xilotepec \\
\hline $\mathrm{BCS}$ & $\mathrm{La} \mathrm{Paz}$ & Los Cabos & & & & & & Zacapoaxtla \\
\hline Camp. & Camp. & Cd. Carmen & & & & & & Zacatlán \\
\hline \multirow[t]{3}{*}{ Coah. } & Saltillo & Acuña & & & & \multirow[t]{2}{*}{ Qro. } & Querétaro & Amealco B. \\
\hline & Torreón & Monclova & \multirow[t]{20}{*}{ Mex. } & \multirow{20}{*}{$\begin{array}{l}\text { Almoloya J. } \\
\text { Atizapán Zar. } \\
\text { Coacalco B. } \\
\text { Chalco } \\
\text { Chimalhuacán } \\
\text { Ecatepec M. } \\
\text { Huixquilucan } \\
\text { Ixtapaluca } \\
\text { Naucalpan J. } \\
\text { Nezahualcoyotl } \\
\text { Nicolás Rom. } \\
\text { La Paz } \\
\text { S. F. Progreso } \\
\text { Tecámac } \\
\text { Texcoco } \\
\text { Tlalnepantla B. } \\
\text { Toluca } \\
\text { Tultitlán } \\
\text { Zinacatepec } \\
\text { Cuauhtitlán } \\
\text { V. Chalco Sol. }\end{array}$} & \multirow{10}{*}{$\begin{array}{l}\text { Atlacomulco } \\
\text { Cuautitlán } \\
\text { Chicoloapa } \\
\text { Ixtlahuaca } \\
\text { Jilotepec } \\
\text { Lerma } \\
\text { Metepec } \\
\text { Tejupilco } \\
\text { Temascalcingo } \\
\text { Temoaya }\end{array}$} & & SJ del Río & El Marqués \\
\hline & Piedras Neg. & \multirow{4}{*}{ Colima } & & & & \multirow[t]{2}{*}{ Q. Roo } & \multirow[t]{2}{*}{ B. Juárez } & Othón Bco. \\
\hline \multirow[t]{3}{*}{ Col. } & \multirow{3}{*}{$\begin{array}{l}\text { Manzanillo } \\
\text { Tecomán }\end{array}$} & & & & & & & Solidaridad \\
\hline & & & & & & SLP & SLP & Cd.Valles \\
\hline & & & & & & & & Matehuala \\
\hline \multirow[t]{3}{*}{ Chis. } & \multirow{3}{*}{$\begin{array}{l}\text { Tapachula } \\
\text { Tuxtla Gtz. } \\
\text { S. C. Casas }\end{array}$} & Comitán & & & & & & Sol. Grac. Schz. \\
\hline & & Ocosingo & & & & Sin. & Ahome & Guasave \\
\hline & & & & & & & Culiacán & \\
\hline Chih. & Aldama & Cuauhtémoc & & & & & Mazatlán & \\
\hline & Allende & & & & & Son. & Cajeme & Guaymas \\
\hline$\overline{\mathrm{DF}}$ & $\begin{array}{l}\text { Azcapotzalco } \\
\text { Coyoacán }\end{array}$ & Milpa Alta & & & $\begin{array}{l}\text { Tenancingo } \\
\text { Tenango Valle. }\end{array}$ & & Hermosillo & $\begin{array}{l}\text { Nogales } \\
\text { SL Río Col. }\end{array}$ \\
\hline & Cuajimalpa & & & & Teoloyucan & Tab. & Cárdenas & Centla \\
\hline & G.A. Madero & & & & Tianguistenco & & Centro & Cunduacá \\
\hline & Iztacalco & & & & Tultepec & & Comalcalco & Macuspana \\
\hline & Iztapalapa & & & & Villa Guerrero & & Huimanguillo & Nacajuca \\
\hline & M. Contreras & & & & Villa Victoria & & & Paraíso \\
\hline & A. Obregón & & & & Zumpango & Tamps. & Cd. Madero & Altamira \\
\hline & Tlahuac & & & & & & Matamoros & Victoria \\
\hline & Tlalpan & & & & & & Nvo. Laredo & \\
\hline & Xochimilco & & & & & & Reynosa & \\
\hline & B. Juárez & & Mich. & Morelia & Apatzingan & & Tampico & \\
\hline & Cuauhtémcoc & & & Uruapán & L. Cárdenas & Tlax. & & Apizaco \\
\hline & M. Hidalgo & & & & Zamora & & & Huamantla \\
\hline & V. Carranza & & Mor. & Cuernavaca & Cuautla & & & Tlaxcala \\
\hline Dgo. & Durango & Gómez Pal. & & & Jiutepec & Ver. & Coatzacoalco & Boca Río \\
\hline Gto. & S. M.Allende & Abasolo & & & Temixco & & Córdova & Coatepec \\
\hline & Celaya & Acámbaro & Nay. & Tepic & & & Xalapa & Cosoleacaque \\
\hline & Dolores Hgo. & Apaseo & $\mathrm{NL}$ & Apodaca & & & Poza Rica & Martínez de la T. \\
\hline & Irapuato & Comonfort & & Gral. Escobedo & & & Veracruz & Minatitlán \\
\hline & León & Guanajuato & & Guadalupe & & & & Orizaba \\
\hline & Salamanca & Pénjamo & & Monterrey & & & & Papantla \\
\hline & Silao & Romita & & S. Nic. Garza & & & & SA Tuxtla \\
\hline & Valle Stgo. & San Felipe & & Sta. Catarina & & & & Tuxpan \\
\hline & & S. F. Rincón & Oax. & Oaxaca & SJB Tuxtepec & Yuc. & Mérida & \\
\hline & & SL de la Paz & Pue. & Puebla & Acajete & Zac. & Fresnillo & \\
\hline & & S. Cruz J.R. & & Tehuacán & Atlixco & & & Zacatecas \\
\hline Gro. & Acapulco & Chilpancingo & & & Huauchinango & & & \\
\hline & & Iguala & & & SM Texmelucan & & & \\
\hline & & & & & SP Cholula & & & \\
\hline & & & & & Tecamachalco & & & \\
\hline
\end{tabular}

\section{Discusión}

La mortalidad por DAN en general, y en particular en menores de 5 años de edad, ha disminuido. Tal descenso se debe en especial a un decremento del grupo de 1 a más años de edad. Esta tendencia la identificaron Peña-Alonso y colaboradores en un estudio de la mortalidad por DAN en el periodo de 1955 a 2003.40 El análisis, de acuerdo con la causa de DAN, revela un leve incremento de los números absolutos y relativos de las defunciones atribuibles a defectos en el SC, aunque convendría analizar si se trata de defectos aislados o bien si son parte de una alteración dismorfológica más compleja.

Es probable, entre las posibles causas del descenso de las muertes por DAN, que sea consecuencia de un mejoramiento de la atención médica especializada de los DAN como reflejo del incremento de la atención 
del parto por personal capacitado, su detección y tratamiento tempranos. Según la Secretaría de Salud, el porcentaje de partos atendidos por personal especializado aumentó de $70 \%$ en el año 2000 a 93.3\% en el $2006 .{ }^{41}$ Los niños con tamizaje neonatal aumentaron de 1437238 en $2003^{42}$ a 1495304 en $2006 .{ }^{41}$ Por otra parte, en México, en las últimas décadas se han efectuado diversos esfuerzos para prevenir, detectar y tratar en forma oportuna los DAN. ${ }^{43-47}$

Es pertinente hacer la distinción entre mortalidad e incidencia de DAN. La primera se altera por muchos factores como la gravedad del padecimiento, la atención médica oportuna y eficaz, la notificación de las defunciones o la calidad del diagnóstico, entre otros, lo que impide equipararla con la incidencia o frecuencia de casos nuevos de DAN. Por lo tanto, los resultados de este trabajo no permiten inferir que la incidencia de DAN experimente una disminución, cautela válida cuando se analizan las cifras de frecuencia de DAN detectadas en los hospitales citados en la introducción de este artículo. ${ }^{16-22}$ Esto suscita la duda acerca de la posibilidad de que dicha frecuencia pudiera elevarse, ya que las cifras de los estudios más antiguos realizados entre los años de 1983 y 1999 tienden a ser menores (1.2, 3.3, 2.5 y $2.31 \%$ ) en comparación con los estudios reportados en años más recientes de 2001 a 2005 (4.0, 3.6, 3.1\%).

Por otra parte, el ejercicio de priorización permitió seleccionar municipios con alta frecuencia de defunciones por DAN en menores de 5 años de edad; pese a ello, no se encontraron ejercicios a nivel municipal publicados en la bibliografía médica con el fin de comparar los presentes resultados. También en este trabajo, como en otros que emplean la mortalidad para reconocer indirectamente áreas de mayor riesgo de incidencia, subyace la inquietud de saber si los municipios prioritarios identificados corresponden en verdad a los de mayor riesgo de productos del embarazo con DAN, preocupación que sólo puede disiparse mediante otro tipo de estudio para conocer la frecuencia de casos nuevos en cada municipio.

Las muertes por DAN en los menores de 5 años se concentran en 206 municipios de alta y muy alta prioridad; en estos municipios pueden realizarse diversas intervenciones, como las que se proponen a continuación: a) estudios sobre calidad diagnóstica de los DAN; la calidad diagnóstica de los DAN es necesaria para un adecuado tratamiento médico y epidemiológico; la genética médica ofrece los principios de dismorfología basados en la etiopatogenia y son de utilidad práctica en la clínica e investigación epidemiológica, lo cual hace posible ubicar el momento del desarrollo prenatal en que ha ocurrido la alteración; con esta clasificación se lograría una mejor caracterización de los DAN y por ende un mejor asesoramiento genético con fines de prevención; b) estudios de georreferenciazación de casos por localidad y domicilio particular, fuentes importantes de contaminación vecinos y la caracterización socioeconómica de los padres de los niños con defectos al nacimiento para la búsqueda de factores sociales adjuntos; c) instrumentar un sistema de vigilancia epidemiológica en coordinación con el programa de atención al recién nacido y niños menores de 5 años, con base en estudios de incidencia de DAN con cierta periodicidad, al momento de la atención del parto en hospitales y el control del crecimiento y desarrollo de los niños durante el primer año de vida y, si es posible, el seguimiento hasta los 5 años de edad; por último, d) analizar si las defunciones fetales tienen comportamiento epidemiológico similar con el empleo del mismo método de priorización.

\section{Referencias}

I. Sá-Carvalho M, Souza-Santos R.Análise de dados epaciais em saúde pública; métodos, problemas, perspectivas. Cad Saúde Pública 2005;2I(2):36I-378.

2. Hernandez-Avila JE, Rodriguez MH, Betanzos-Reyes AF, Danis-Lozano R, Mendez-Galvan JF, Tapia-Conyer R, et al. Determinant factors for malaria transmission on the coast of Oaxaca State, the main residual transmission focus in Mexico. Salud Publica Mex 2006;48(5):405-4I7.

3. Hijar-Medina MC. Utilidad del análisis geográfico en el estudio de las muertes por atropellamiento. Salud Publica Mex 2000;42(3): I88-193.

4. Barquera S, Tolentino L. Geografía de las enfermedades asociadas con la nutrición en México. Pap Pobl 2005;43:|33-149.

5. Hernández-Ávila JE, Rodríguez MH, Rodríguez NE, Santos R, Morales E, Cruz C, et al. Cobertura geográfica del sistema mexicano de salud y análisis espacial de la utilización de hospitales generales de la Secretaría de Salud en 1998. Salud Publica Mex 2002;44(6):519-523.

6. Bailey TC. Spatial statistical methods in health. Cad Saude Publica 2001;17(5): 1083-1098.

7. Lantada-Zarzosa N, Núñez-Andrade MA. Sistemas de Información geográfica. Prácticas en ArcView. Bogotá:Alfaomega/Ediciones UPC, 2006. 8. Martínez-Piedra R, Loyola-Elizondo E,Vidaurre-Arenas M, NájeraAguilar P. Paquetes de programas de mapeo y análisis espacial en epidemiología y salud pública. Bol Epidemiol 2004;25(4). [Consultado 2009 mar 19]. Disponible en: http://www.paho.org/spanish/dd/ais/ be_v25n4-soft_sig_sp.htm

9. Elliot P,Wartenberg D. Spatial epidemiology: current appproaches and future challenges. Environ Health Persp 2004; I I2(9):998- 1006.

I0.Ticket K, Peral M. Multilevel analises of neighbourhood socioeconomic context and health outcomes: a critical review. J Epidemiol Community Health 200I; 155:III-I22.

II. Norma Oficial Mexicana NOM-034-SSA2-2002, para la Prevención y Control de los Defectos al Nacimiento. [Consultado 2009 abr 02]. Disponible en: http://www.salud.gob.mx/unidades/cdi/nom/034ssa202.html 12. Secretaría de Salud. Mortalidad 2000-2005 y 2007. [Consultado 2009 abr 02]. Disponible en: www.sinais.salud.gob.mx.

13. Emery and Rimoin's. Principles and practice of medical genetics. Chap. 17 Human malformations. Clayton-Smith J, Donnai D, 488-500. 4rd ed. vol. I. Rimoin DL, Connor JM, Pyeritz RE, Korf BR, (eds). USA-UK: Elsevier Science, 2002.

14. Jorde L, Carey J, Bamshad M,Withe R. Genética médica. Madrid: Elsevier, 2005: 305-324. 
15. Morales J], Luna L, Mutchinick O. Epidemiología de las malformaciones congénitas. En: Guízar-Vázquez. Genética clínica. Diagnóstico y manejo de las enfermedades hereditarias. México, D.F. - Santa Fe de Bogotá: El Manual Moderno, 200I.

16. Hernández A, Corona-Rivera E, Martínez-Basalo C, Aguirre-Negrete G, Fonseca S, Cantú JM. Factores prenatales y defectos congénitos en una población de 779I recién nacidos consecutivos. Bol Med Hosp Infant Mex 1983; 40(7):363-366.

17. Canún-Serrano S, Zafra-de la Rosa G. Detección de malformaciones congénitas externas. Incidencia en 3,283 recién nacidos consecutivos. Bol Med Hosp Infant Mex 1984;4I (I) 2I-24.

18. Canún-Serrano S, Saavedra-Ontiveros D, Chavira ES, Andrade TF. Malformaciones congénitas en diferentes etapas del desarrollo intrauterino. Ginec y Obstet (Méx) 1990; 58: I-4.

19. Arredondo G, Rodríguez-Bonito R, Treviño-Alanís MG,Arreola B, Astudillo-Castillo G, Russildi JM. Malformaciones congénitas en recién nacidos vivos. Bol Med Hosp Infant Mex 1990;47(12) 822-827.

20. Fernández-Carrasco I, Hernández-Valle A. Defectos al nacimiento encontrados en la Clínica de Especialidades de la Mujer, periodo 20012004. Rev Sanid Milit Mex 2006; 60(4):230-239.

2I. Aguinaga M, Llano I, Báez R, Hernández C, Castro J, et al. Análisis y resultados clinicocitogenéticos de fetos y recién nacidos con alteraciones cromosómicas durante un año en el Instituto Nacional de Perinatología. Perinatol Reprod Hum 2005;19(2):94-105.

22.Vega-Valdés A,Vizzuett Martínez R. Frecuencia de malformaciones congénitas en un hospital general de tercer nivel. Rev Mex Pedia 2005;72(2):70-73.

23. Rivera-Rueda M, Hernández-Trejo M, Hernández-Peláez G, Llano-Rivas I, Di Castro-Stringher P, et al. Análisis de la mortalidad neonatal precoz en el Instituto Nacional de Perinatología (1999-200I). Perinatol Reprod Hum 2005; 19(I):|3-2I.

24. Hernández-Trejo M, Llano-Rivas I, Rivera-Rueda A,Aguinaga-Ríos M, Mayen-Molina D. Mortalidad perinatal por defectos estructurales congénitos: un estudio de sitio. Perinatol Reprod Hum 2007; 2I(3): I25-I32. 25. Nussbaum RL, Mclnnes RR,Willarde HE, Boerkoel CF III. Thompson \& Thompson genetics in medicine. 6th ed. 200I USA. Boerkel CF III. Clinical case studies illustrating genetic principles. Achondroplasia (FGFR3 mutation) autosomal dominant. Achondroplasia. Francomano CA. Risk to family members. Parents of a proband. [Consultado 2009 julio 3]. Disponible en: http://www.geneclinics.org.

26. Guízar-Vázquez. Genética clínica. Diagnóstico y manejo de las enfermedades hereditarias. México, D.F.-Santa Fe de Bogotá: El Manual Moderno, 200I.

27. Secretaría de Salud. Tablas dinámicas de mortalidad. [Consultado 2008 julio 15]. www.sinais.salud.gob.mx.

28. World Health Organization. International Statistical Classification of Diseases and Related Health Problems. I0th Revision.Version 2007. Disponible en: [Consultado 2009 marzo 26]. http://apps.who.int/ classifications/apps/icd/icdIOonline.

29. Instituto Nacional de Estadística, Geografía e Informática. Datos municipales digitalizados. [Consultado 2008 julio I5]. Disponible en: www. inegi.gob.mx.

30. Secretaría de Salud. Proyecciones de Población de CONAPO 20002050. [Consultado 2008 julio I5]. Disponible en: www.salud.gob.mx. 31. Instituto Nacional de Estadística, Geografía e Informática. Marco Geoestadístico Municipal 2005. México: INEGI.
32. Silva-Aycaguer C, Benavides-Rodríguez A,Vidal-Rodeiro CL. Análisis espacial de la mortalidad en áreas geográficas pequeñas: el enfoque bayesiano. Rev Cub Salud Publica 2003;29(4):3।4-322.

33. Loyola E, Castillo C, Nájera P,Vidaurre M, Mujica O, Martínez R. Los sistemas de información geográfica como herramienta para monitorear las desigualdades de salud. Rev Panam Salud Pública 2002;12(6):415-428. 34. Prates-Melo EC, Sá-Carvalho M,Travassos C. Distribucao espacial da mortalidade por infarto agudo do miocárdio no Municipio do Rio de Janeiro, Brasil. Cad Saude Publica 2006;22(6):I225-I236.

35. Prates-da-Fonseca H.Vulnerabilidade socioambiental na metrópoli paulistana: uma análise sociodemográfica das situacoes de sobreposicao espacial de problemas e riscos sociais e ambientáis. R Bras Est Pop 2006;23(I):43-59.

36. Lapa TM, Militao-de-Alburquerque MF, Sá-Carvalho M.Análise da demanda de casos de hanseniase aos servicos de saúde a través do uso de técnicas de análise espacial. Cad Saude Publica 2006;22(I2):2575-2583. 37. Barrera R, Delgado N, Jiménez M,Villalobos I, Romero I. Estratificación de una ciudad hiperendémica en dengue hemorrágico. Rev Panam Salud Publica 2000;8(4):225-233.

38. Barcillos Chr,Weide-Acosta LM, Pedroso-Lisboa E,Varnieri-Brito MR, Flores R. Estimativa da prevalencia de HIV em gestantes por análise espacial, Porto Alegre, RS. Cad Saude Publica 2006;40(5):928-930. 39. Barcillos Chr, Lammerhirt CB,Almeida MA, Dos-Santos E. Distribucao espacial da leptospirose no Rio Grande do Sul, Brasil: recuperando a ecologia dos estudos ecológicos. Cad Saude Publica 2003; I9(5): 1283-1292. 40. Peña-Alonso R, Venegas C, Lozano R, Kofman-Alfaro S, Queipo G. Mortalidad por defectos al nacimiento. Bol Med Hosp Infant Mex 2005;62:294-304.

4I. Secretaría de Salud. Situación de Salud en México. Indicadores básicos, 2000-2006. [Consultado 2009 mar 26]. Disponible en: www.sinais.salud. gob.mx/publicaciones/index.html.

42. Torres-Sepúlveda MR, Martínez-de-Villarreal LE, Esmer C, GonzálezAlanís R, Ruiz-Herrera C, Sánchez-Peña A, et al. Tamiz metabólico neonatal por espectrometría de masas en tándem: dos años de experiencia en Nuevo León, México. Salud Publica Mex 2008;50:200-206.

43. Secretaría de Salud. Boletín de Información Estadística. Volumen III. Servicios otorgados y Programas Sustantivos, 2003-2006. [Consultado 2009 mar 26]. Disponible en: www.sinais.salud.gob.mx/publicaciones/ index.html.

44. Leis MT, Hernández E, Maya D, Garza-Morales S, García-Cavazos R, DíazCáceres AC. Evaluación multidisciplinaria del feto con hidrocefalia: protocolo de estudio y resultados. Ginecol Obstet Mex 1996;64(4):154-160. 45. UNICEF/SSa-Distrito Federal. Propuestas de política pública para atender las necesidades de niños y niñas con discapacidad en zonas marginadas del Distrito Federal. [Consultado 2009 mar 25]. Disponible en:www.unicef.org/mexico/spanish/mx_resources_reto_inclusion_ estudio_discapacidad.pdf.

46. Secretaría de Salud/Dirección General de Salud Reproductiva. Prevención, detección, manejo y seguimiento de los defectos al nacimiento. México: SSa, 2002.

47. Secretaría de Salud/Dirección General de Epidemiología. Manual para la vigilancia epidemiológica de los defectos del Tubo Neural. México: SSa, 1999. 